\section{Aloimunização RhD em gestantes no Estado do Rio de Janeiro, Brasil: perspectivas e desafios}

\author{
$\mathrm{RhD}$ alloimmunization in pregnant women in \\ Rio de Janeiro State, Brazil: perspectives \\ and challenges
}

\begin{abstract}
Aloinmunización RhD en gestantes del estado de Río de Janeiro, Brasil: perspectivas y desafíos
\end{abstract}

Ana Heloisa Nascimento Beserra ${ }^{1}$ Elizabeth Artmann 2

Maria Cristina Pessoa dos Santos 1

\section{Resumo}

O estudo mostra resultados da pesquisa sobre a persistência da aloimunização RhD nas gestantes atendidas na rede pública do Rio de Janeiro, Brasil, por meio de análises de prontuários e de entrevistas com gestores, profissionais de saúde e gestantes. Analisaram-se 289 prontuários de gestantes RhD negativas atendidas entre 2004 a 2012 no Centro de Referência Estadual. Realizaram-se entrevistas com 15 atores sociais, individualmente. As entrevistas apresentaram fatores que contribuíram para a persistência do problema como: perda de reestruturação do Programa de Prevenção da Aloimunização RhD (PPARhD); ausência de um sistema de monitoramento e avaliação do PPARhD; a baixa articulação da rede de referência e contrarreferência de atendimento pré-natal; a capacitação insuficiente dos profissionais de saúde que atendem a população em risco e a insuficiente divulgação do programa de prevenção da aloimunização. Esses nós críticos apontam a necessidade da Secretaria Estadual de Saúde voltar a priorizar o programa de prevenção de aloimunização e investir na articulação da rede de assistência. O estudo mostrou que o problema é multicausal e que enfrentá-lo exige ações interdisciplinares e complexas relacionadas ao atendimento integral no pré-natal e à atenção à saúde da mulher.

Isoimunização Rh; Gestantes; Saúde da Mulher; Planejamento em Saúde

\author{
${ }^{1}$ Instituto Nacional de Saúde \\ da Mulher, da Criança e \\ do Adolescente Fernandes \\ Figueira, Fundação Oswaldo \\ Cruz, Rio de Janeiro, Brasil. \\ 2 Escola Nacional de Saúde \\ Pública Sergio Arouca, \\ Fundação Oswaldo Cruz \\ Rio de Janeiro, Brasil. \\ Correspondência \\ A. H. N. Beserra \\ Instituto Nacional de Saúde \\ da Mulher, da Criança e \\ do Adolescente Fernandes \\ Figueira, Fundação Oswaldo \\ Cruz. \\ Av. Rui Barbosa 716 \\ Rio de Janeiro, $R J$ \\ 22250-020, Brasil. \\ anaheloisa@superig.com.br
}




\section{Introdução}

No cenário internacional, as taxas de mortalidade materna (TMM) e as taxas de mortalidade neonatal (TMN) estão em queda, assim como no Brasil, embora em menor proporção 1,2. No Estado do Rio de Janeiro, a implantação da Rede Cegonha, em 2011, vem reduzindo esses índices, ainda que não na mesma proporção ${ }^{2}$, pois encontra um conjunto de problemas na qualidade de atendimento de pré-natal ${ }^{3}$. A aloimunização RhD é elencada como um dos cuidados pelo Manual Técnico de Gestação de Alto Risco, considerada um problema evitável, pela administração da imunoglobulina anti-RhD em gestantes RhD negativo em dose e momento adequados 4 . Trabalhos internacionais 5,6 também apontaram a preocupação com políticas e ações pertinentes de prevenção da aloimunização RhD e seus desdobramentos fetais/neonatais. No Rio de Janeiro, instituiu-se em 2003 o Programa de Profilaxia da Aloimunização RhD, objetivando proteger as mulheres RhD negativo, da aloimunização durante o ciclo gravídico puerperal e reduzir a morbidade e mortalidade perinatal por incompatibilidade RhD. Esse programa propõe ações de controle da aquisição e distribuição da imunoglobulina anti-RhD para o SUS; de divulgação e treinamento do protocolo da sua utilização terapêutica entre os profissionais de saúde e na divulgação do risco da aloimunização entre gestantes. Este estudo justifica-se pela necessidade de avanços na qualidade da assistência pré-natal e teve como objetivo verificar o porquê e onde ocorre o problema da aloimunização RhD no Estado do Rio de Janeiro e discutir propostas para seu enfrentamento.

\section{Métodos}

O estudo explorou dados de prontuários de gestantes RhD aloimunizadas encaminhadas e atendidas no Centro de Referência Estadual do Instituto Nacional de Saúde da Mulher, da Criança e do Adolescente Fernandes Figueira, Fundação Oswaldo Cruz (IFF/Fiocruz), no Rio de Janeiro, no período de 2004 a 2012. Descreveu-se o perfil por categorias referentes aos dados do histórico sociodemográfico, reprodutivo e gestação atual. $\mathrm{O}$ armazenamento dos dados foi feito por meio do programa Excel (Microsoft Corp., Estados Unidos) e a análise, pelo teste qui-quadrado e risco estimado, de todas as categorias buscadas, utilizando o software SPSS 16.0 (SPSS Inc., Chicago, Estados Unidos).

Foram ainda realizadas entrevistas com 15 atores que participaram desse cenário. Os cri- térios de seleção dos entrevistados foram: (a) gestores pela sua contribuição na elaboração do Programa de Profilaxia da Aloimunização RhD (PPARhD); (b) profissionais de saúde que trabalham no Centro de Referência, os que assistem municípios do Rio de Janeiro e encaminham gestantes ao IFF; (c) gestantes com RhD negativas aloimunizadas acompanhadas no centro de referência durante o período do estudo.

Baseado nos conceitos do Planejamento Estratégico Situacional (PES) 7, definiu-se como problema a persistência da aloimunização $\mathrm{RhD}$. Construiu-se uma rede explicativa com base nos resultados dos prontuários e na análise das entrevistas. Selecionaram-se os nós críticos (NC), ou seja, os nós alvos de intervenções, segundo os critérios utilizados, baseados no PES 7,8: alto impacto sobre o problema; centros práticos e efetivos de enfrentamento e oportunidade política. O estudo foi aprovado pelo Comitê de Ética em Pesquisa do IFF, parecer no 1425/14.

\section{Resultados e discussão}

A análise dos 289 prontuários de gestantes RhD negativas mostrou escolaridade da maioria entre alfabetizada ou 1o grau; os municípios de encaminhamento mais frequentes para a referência foram Nova Iguaçu e São Gonçalo; origem na rede de serviço pública; em sua 2a ou 3 a gestação; chegada com maior frequência no centro de referência no terceiro trimestre de gestação (Tabela 1), evidenciando uma lacuna na conversação das redes de serviço. A chegada tardia das gestantes, um nó explicativo importante, revela problemas no atendimento relacionados aos outros nós como a inadequada capacitação dos profissionais, levando ao não diagnóstico pertinente em tempo e, consequentemente, levando à perda da oportunidade de uso da imunoglobulina em tempo adequado; dificuldades na rede de referência e contrarreferência; peregrinação desnecessária da paciente, com consequências irreversíveis como óbito fetal/neonatal (Figura 1).

Em praticamente todas as categorias, predominou o não uso da imunoglobulina em evento anterior, seja gravidez ou aborto. A análise estatística não revelou diferença significativa em nenhuma das categorias estudadas. Não aprofundamos a interpretação desse resultado, considerando que o " $n$ " limitado não permite maiores generalizações. Outro problema encontrado na busca foi que muitos não relatavam informações e registros importantes como o uso ou não da imunoglobulina em evento anterior.

Os resultados das entrevistas permitiram explorar as causas do problema da persistên- 
Perfil das gestantes aloimunizadas atendidas no Instituto Nacional de Saúde da Mulher, da Criança e do Adolescente

Fernandes Figueira (IFF) entre 2004 a 2012 e a informação do uso da imunoglobulina anti-RhD (lgGRhD) em evento anterior.

\begin{tabular}{|c|c|c|c|}
\hline \multirow[t]{2}{*}{ Categorias/Gestantes } & \multicolumn{2}{|c|}{ Uso anterior de IgGRhD } & \multirow[t]{2}{*}{ Total } \\
\hline & $\operatorname{Sim}(n)$ & Não (n) & \\
\hline \multicolumn{4}{|l|}{ Idade (anos) } \\
\hline$\leq 29$ & 58 & 56 & 114 \\
\hline $30-39$ & 53 & 79 & 132 \\
\hline$\geq 40$ & 6 & 6 & 12 \\
\hline \multicolumn{4}{|l|}{ Escolaridade } \\
\hline Analfabeta & 1 & 1 & 2 \\
\hline Alfabetizada ou 1o grau & 48 & 61 & 109 \\
\hline 2o grau & 42 & 52 & 94 \\
\hline 3o grau & 6 & 9 & 15 \\
\hline \multicolumn{4}{|l|}{ Moradia } \\
\hline Rio de Janeiro & 75 & 80 & 155 \\
\hline Duque de Caxias & 2 & 10 & 12 \\
\hline Nova Iguaçu & 3 & 11 & 14 \\
\hline São João de Meriti & 3 & 5 & 8 \\
\hline São Gonçalo & 3 & 12 & 15 \\
\hline Outros & 31 & 23 & 54 \\
\hline \multicolumn{4}{|l|}{ Origem } \\
\hline Pública & 49 & 53 & 102 \\
\hline Particular & 10 & 13 & 23 \\
\hline \multicolumn{4}{|l|}{ Número de gestações } \\
\hline 1 & 0 & 5 & 5 \\
\hline $2-3$ & 53 & 77 & 130 \\
\hline Mais de 4 & 64 & 59 & 123 \\
\hline \multicolumn{4}{|c|}{ Idade gestacional de chegada } \\
\hline 1으 trimestre & 9 & 14 & 23 \\
\hline 2o trimestre & 42 & 58 & 100 \\
\hline 3o trimestre & 50 & 55 & 105 \\
\hline
\end{tabular}

Fonte: prontuários de gestantes RhD negativas atendidas no IFF de 2004 a 2012.

cia da aloimunização expressas no fluxograma situacional (Figura 1). Utilizaram-se os critérios do PES 7,8 referidos acima, para apontar os nós críticos do problema.

$\mathrm{Na}$ rede explicativa, foram identificados os seguintes nós críticos: NC1-ausência de um sistema de monitoramento/avaliação; NC2-baixa articulação da rede de atendimento pré-natal; NC3-divulgação insuficiente do PPARhD; e NC4-perda de estruturação do PPARhD. Embora o nó explicativo "baixa prioridade na gestão da Secretaria Estadual de Saúde (SES) e das Secretarias Municipais de Saúde (SMS)" tenha sido entendido como de alto impacto pelos gestores entrevistados, não se constitui como centro de ação, e deve ser atingido indiretamente mediante intervenção nos NC1, NC2 e NC3. A baixa articulação da rede exige envolvimento de diferentes atores (gestores e profissionais) da SES, da SMS e do IFF na busca por maior governabilidade em seu enfrentamento.

Nas entrevistas com profissionais e gestores, foi realçada a necessidade de educação continuada dos profissionais de saúde, um fluxo frequente de informação entre os serviços, destacando a importância do uso da imunoglobulina para a população de risco. Os achados desse estudo corroboram os encontrados em vários outros 5,6,9 como a existência de falhas na aplicação dos protocolos de profilaxia, a omissão ou a administração tardia, dose incorreta ou insuficiente, o uso inadequado em pacientes $\mathrm{RhD}$ positivas ou aloimunizadas, mães com recémnascidos RhD negativos, além dos erros de manuseios e estocagem da imunoglobulina. 
Figura 1

Rede explicativa da persistência da aloimunização RhD em gestantes no Estado do Rio de Janeiro, Brasil.

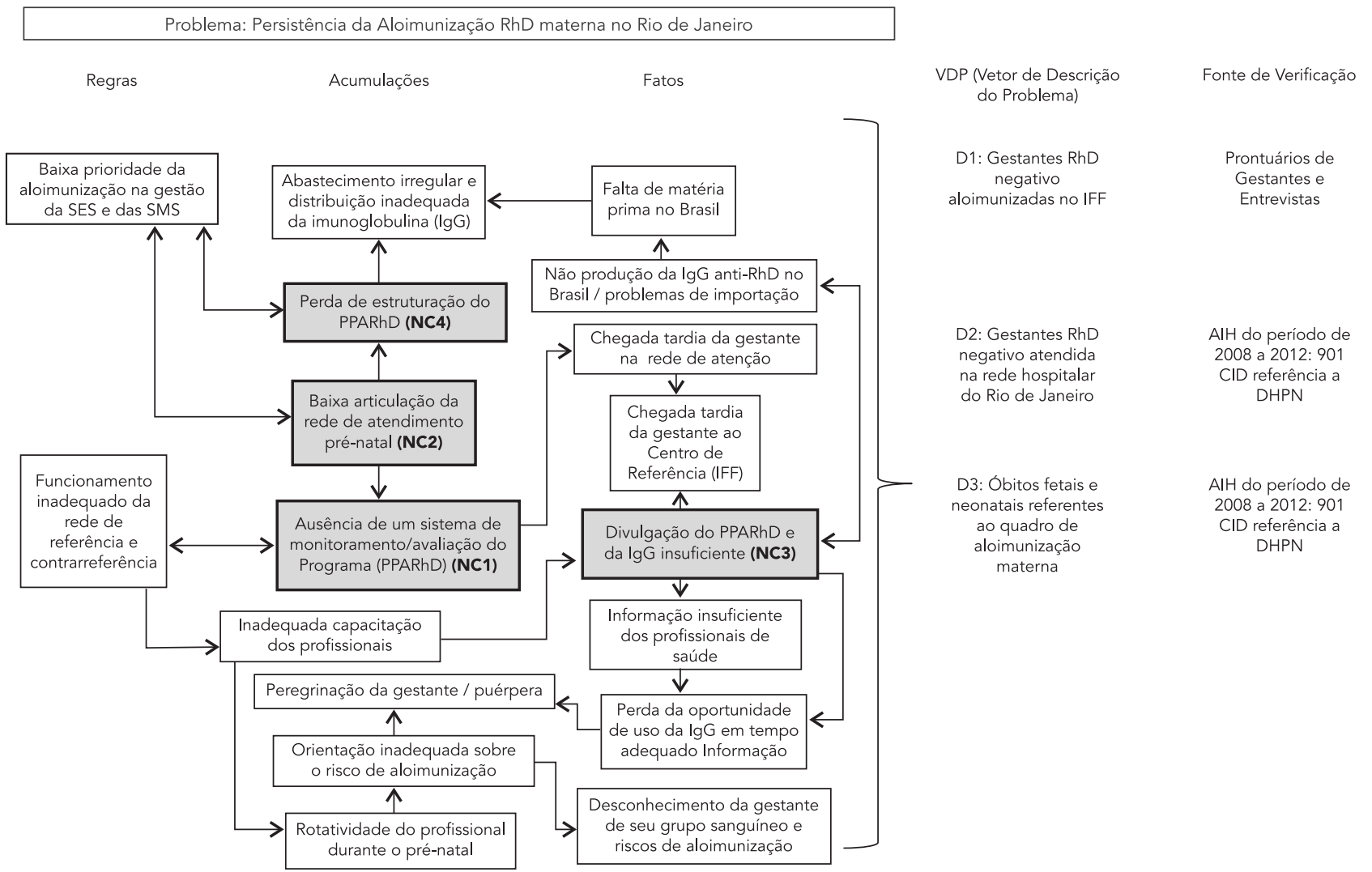

AlH: Autorização de Internação Hospitalar; CID: Classificação Internacional de Doenças; DHPN: doença hemolítica perinatal; IFF: Instituto Nacional de Saúde da Mulher, da Criança e do Adolescente Fernandes Figueira; PPARhD: Programa Estadual de Profilaxia de Aloimunização RhD; NC: nó crítico; SES: Secretaria Estadual de Saúde; SMS: Secretaria Municipal da Saúde.

Fonte: Pesquisa sobre Persistência da Aloimunização RhD em Gestantes no Estado do Rio de Janeiro, Brasil.

As dificuldades de acesso aos serviços de saúde, a necessidade de um pré-natal especializado para as mulheres aloimunizadas e estruturas complexas para o acompanhamento das gestantes e recém-nascidos, a capacitação das equipes nas redes ambulatorial e hospitalar, para possibilitar um diagnóstico rápido e correto 9 , foram questões também apontadas nas entrevistas realizadas. Alguns de tais aspectos estão imbricados com questões fora da governabilidade dos atores envolvidos diretamente e demandariam articulação com setores fora do sistema da saúde, como o setor de transporte e o de educação. Por isso, além do papel fundamental da SES, outros atores e setores deveriam participar na busca de enfrentamento do problema.

Ações preventivas e de intervenção são necessárias para melhorar a qualidade de atenção ao cuidado na assistência ao pré-natal 10 . No caso da aloimunização $\mathrm{RhD}$, as medidas necessárias seriam a identificação laboratorial correta das pacientes de risco (Rh negativas), a aplicação, quando possível, da imunoglobulina na $28^{\mathrm{a}}$ semana de gestação e a garantia da prescrição e aplicação adequada da imunoglobulina antiRhD no período puerperal 4.

A "baixa articulação da rede de referência e contrarreferência" foi apontada como um dos nós críticos que demandam intervenção, assim como os outros três e é importante destacar que todos têm alto impacto sobre o problema, não sendo possível resolver a situação de forma parcial. Contudo, a atual situação da rede de atenção no Rio de Janeiro, bastante precária, não deve paralisar as ações, pelo contrário. Assim, observa-se que uma revalorização do PPARhD (NC4) seria 
fundamental juntamente como um sistema de monitoramento específico que funcionasse como acompanhamento e "vigilância" das gestantes de risco, evitando dessa forma, portanto, desfechos fetais graves. Logo, um plano de enfrentamento da situação deveria ter em conta uma boa análise de viabilidade e investir fortemente em ações de impacto mais imediato, como aquelas relacionadas no PPARhD, e ao mesmo tempo, em outras de mais longo prazo, como a melhoria de articulação da rede de atendimento pré-natal.

Buscou-se neste artigo priorizar e apontar alguns dos nós críticos do problema estudado. $\mathrm{O}$ grande desafio se revelou alcançar maior articulação da rede de atendimento pré-natal. O panorama geral sobre a assistência pré-natal no Brasil mostra cobertura praticamente universal, porém persistem desigualdades regionais e sociais no acesso a um cuidado adequado 10,11. Ainda que a persistência da aloimunização não se expresse de forma tão contundente quantitativamente como outros agravos, por ter um percentual menor de Rh negativo na população brasileira, e por isso se torna um problema menos visível, as consequências são muito graves com desfechos muitas vezes irreversíveis. Todavia, há formas de prevenção e tecnologia que permitem enfrentar adequadamente o problema, mas que exigem ações interdisciplinares e complexas relacionadas ao atendimento integral no pré-natal e à atenção à saúde da mulher.

\section{Colaboradores}

A. H. N. Beserra participou na concepção, elaboração, pesquisa, escrita e revisão do artigo. E. Artmann e M. C. P. Santos participaram na concepção, elaboração, escrita e revisão do artigo.

\section{Agradecimentos}

Ao pessoal do arquivo médico, ao Prof. Dr. Saint Clair dos Santos Gomes Junior pela contribuição na parte estatística, à Profa. Dra. Cynthia Magluta, aos gestores, aos profissionais de saúde e às gestantes participantes que tornaram este estudo possível. À Capes pelo apoio recebido relativo ao Programa de Apoio ao Mestrado Acadêmico no IFF/Fiocruz no estado do Rio de Janeiro.

\section{Referências}

1. Victora CG, Aquino EML, Leal MC, Monteiro CA, Barros FC, Szwarcwald CL. Maternal and child health in Brazil: progress and challenges. Lancet 2011; 377:1863-76.

2. Portal Brasil. Taxa de mortalidade infantil no País cai $77 \%$ desde 1990. http://www.brasil.gov.br/sau de/2013/09/taxa-de-mortalidade-infantil-no-paiscai-77-desde-1990 (acessado em 05/Mai/2016).

3. Subsecretaria de Atenção à Saúde, Secretaria de Estado de Saúde do Rio de Janeiro. Projeto Qualisus, 2012. http://www.informacaoemsaude.rj.gov. br/docman/atencao-a-saude/7978-mapa-da-sau de-metroi-qualisus-final-060112/file.html (acessado em 05/Mai/2016).

4. Departamento de Atenção Básica, Secretaria de Atenção à Saúde, Ministério da Saúde. Doença hemolítica perinatal. Atenção ao pré-natal de baixo risco. Brasília: Ministério da Saúde; 2012. (Cadernos de Atenção Básica).

5. Bolton-Maggs PHB, Davies T, Poles D, Cohen H. Erros in anti-D immunoglobulin administration: retrospective analysis of 15 years of reports to the UK confidential haemovigilance scheme. BJOG 2013; 120:873-8.

6. Velati C, Reali G, Biffoni F, Bonomo P, Angiolino A, Morelati F, et al. A survey of the current use of anti-D immunoprophylaxis and the incidence of haemolytic disease of the newborn in Italy. Blood Transfus 2007; 5:7-14.

7. Matus C. El PES en la práctica. Caracas: Fundación Altadir; 1994.

8. Rivera FJU, Artmann E. Planejamento e gestão em saúde: conceitos, história e propostas. 1ạ Ed. Rio de Janeiro: Editora Fiocruz: 2012. (Coleção Temas em Saúde). 
9. Sá CAM. Doença hemolítica perinatal RhD. Um problema de saúde pública no Brasil [Tese de Doutorado]. Rio de Janeiro: Instituto Nacional de Saúde da Mulher, da Criança e do Adolescente Fernandes Figueira, Fundação Oswaldo Cruz; 2013.

10. Viellas EF, Domingues RMSM, Dias MAB, Gama SGN, Theme Filha MM, Costa JV, et al. Assistência pré-natal no Brasil. Cad Saúde Pública 2014; 30 Suppl:S85-S100.

\section{Abstract}

This study shows research results on the persistence of RhD alloimmunization in pregnant women seen in the public healthcare network in Rio de Janeiro State, Brazil, through patient file analysis and interviews with administrators, health professionals, and patients. We analyzed 289 patient files of RhD-negative pregnant women seen from 2004 to 2012 at the State Reference Center. Individual interviews were held with 15 individuals. The interviews revealed factors contributing to persistence of the problem, such as: loss of restructuring of the Program for the Prevention of RhD Alloimmunization (PPARhD); lack of a system for monitoring the Program; low linkage between the referral and counter-referral network during prenatal care; insufficient training of the health professionals treating pregnant women at risk; and insufficient visibility of the program for RhD alloimmunization prevention. These critical points highlight the need for the State Health Department to reinforce prioritization of the program for alloimmunization prevention and invest in referral and counter-referral in the healthcare network. The study showed that the problem has multiple causes and requires interdisciplinary and complex measures related to comprehensive prenatal care.

Rh Isoimmunization; Pregnant Women; Women's Health; Health Planning
11. Domingues RMSM, Viellas EF, Dias MAB, Torres JA, Theme Filha MM, Gama SGN, Leal MC. Adequação da assistência pré-natal segundo as características maternas no Brasil. Rev Panam Salud Pública 2015; 37:140-7.

\section{Resumen}

El estudio muestra resultados de la investigación sobre la persistencia de la aloinmunización RhD en las gestantes atendidas en la red pública de Río de Janeiro, Brasil, mediante análisis de historiales médicos y de entrevistas con gestores, profesionales de salud y gestantes. Se analizaron 289 historiales clínicos de gestantes RhD negativas, atendidas de 2004 a 2012, en el Centro de Referencia Estatal. Se realizaron entrevistas con 15 actores sociales, individualmente. Las entrevistas presentaron factores que contribuyeron a la persistencia del problema como: pérdida de reestructuración del Programa de Prevención de la Aloinmunización RhD (PPARhD); ausencia de un sistema de monitoreo y evaluación del PPARhD; la baja coordinación de la red de referencia y contrarreferencia de atención prenatal; la capacitación insuficiente de los profesionales de salud que atienden a la población en riesgo y la insuficiente divulgación del programa de prevención de la aloinmunización. Esos nudos críticos apuntan la necesidad de la Secretaría Estatal de Salud de volver a priorizar el programa de prevención de aloinmunización e invertir en la coordinación efectiva de la red de asistencia. El estudio mostró que el problema es multicausal y que enfrentarlo exige acciones interdisciplinares y complejas, relacionadas con la atención integral durante el periodo prenatal y la atención a la salud de la mujer.

Isoinmunización Rh; Mujeres Embarazadas; Salud de la Mujer; Planificación en Salud
Recebido em 13/Jan/2016

Versão final reapresentada em 26/Jul/2016

Aprovado em 22/Set/2016 